\title{
On the Artistic Features of Zhang Qinghui's Oil Paintings-Take<The Past to Be Taken Away by The Clouds> For Example
}

\author{
Fan Du \\ Nanchong Vocational and Technical College Department of Humanities and Arts Nanchong City, \\ Sichuan Province 637131, China.
}

\begin{abstract}
As a new generation painter, Zhang Qinghui's oil painting has many unique characteristics in painting techniques. His representative work < The past to be taken away by the clouds $>$ is a representative of Zhang Qinghui's profound painting attainments. Based on this, this article takes "the past events taken by the clouds" as an example to analyze the artistic features of the Qing Hui oil painting, so that we can better understand the cultural connotation and profound meaning of Zhang Qinghui's painting.
\end{abstract}

Key Words: Zhang Qinghui; oil paintings; artistic features.

\section{论张庆慧油画作品的艺术特点 一以《被云带走的往事》为例}

\section{杜凡}

南充职业技术学院, 四川南充 637131, 中国.

摘 要：张庆慧作为新生代画家，其油画作品在绘画技巧上有着于众不同的独特之处。其代 表作《被云带走的往事》是可谓代表了张庆慧深厚绘画造诣, 本文基于此以《被云带走的往 事》为例, 对庆慧油画作品的艺术特点进行分析, 以便让我们更好了解张庆慧绘画文化内涵 和深刻寓意。

关键词: 张庆慧；油画作品；艺术特点.

\section{1. 引言}

张庆慧, 汉族, 女画家。2004 年毕业于西安工业学院（现西安工业大学）艺术系。毕业后任 教于山东服装职业学院, 现于北京从事专业油画创作。油画作品曾多次参加艺术展览并被 国内外藏家及美术馆等专业机构收藏。

一个画家要创造出具有生命力和艺术感的作品, 就不能用固有的思维和眼光来展现现实生活, 要通过时代脉搏的体现来凸显现实的民族生活。其次, 把具象写实作为油画外在风貌的表现 手法，运用兼容表现与象征等诸多因素，能将真实、深刻的思想内容直接传达给观众，从而 引起人们心灵的共鸣。再次, 现代主义、后现代主义等艺术潮流, 要相互交融、相互补充, 以便形成多元化的艺术形式。从当前中国美术发展的现状来看, 深入生活的现实主义绘画手 法才是大众所能接受的艺术形式, 张庆慧油画作品《被云带走的往事》一方面根植于中国传 统绘画技巧, 另一方面将西方印象画的绘画风格融入其中, 下面就这幅绘画的艺术特点进行 逐一论述。

\section{2. 优雅的孤独格调}

张庆慧以自己的方式探寻心灵归属的表达。画家以一种独具个人化的造型和图式描绘着自己 内心的思绪, 直击心灵。画面中没有当代艺术风格中常见的张扬和喧闹, 有的却是一份沉静 和淡然, 独具一种优雅的孤独格调。 
张庆慧的作品具有波谱化的视觉趣味, 有着较为明确和简练的色相关系, 看起来像是怀旧时 尚海报中的丝网效果。早期作品中，她有意地从欧美的城市景观和时尚海报中去获取创作资 源，稍后的作品则以 “中国女孩” 作为主要题材，似乎是将 “自我” 在画面中做镜像化呈现。 但从 2012 年开始, 这种活泼、时尚的风格有了明显的变化, 她开始使用较为单纯的褐色调子 进行创作。这种色调是西方学院派绘画中非常主流的视觉趣味, 在 17-19 世纪的作品中, 甚 至连画中物象的固有色都会被罩上一层 “酱油色”，这种趣味直到印象派的出现才被真正打 破。尽管如此, 在不少现代艺术家的创作中仍然常见。张庆慧的某些作品倒容易让人想起美 国画家怀斯作品中的那种哀伤感和被遗弃感。

在 2014 年创作的作品《被云带走的往事》。冷寂的荒原, 一望无际, 看不到一丝绿色, 老旧 的单人沙发孤零零地诉说着自己的故事, 但我们却无法听清。她有意地为画面做了减法, 除 了这件单人沙发, 我们只能看见那似乎已经无法孕育生命的土地。这副画很容易让人想起梵 高的画作一一《农鞋》, 同样是破旧的人造物, 同样是荒凉的背景。旧沙发与农鞋一样, 它 们是 “人” 的表征, 因为留下了人的痕迹而具有着文化意义。有了它, 观者可以从中去感知 历史, 去挖掘隐藏的意义。作品的高明之处就在于, 它并不清晰地呈现意义, 而是给人留下 无穷的想象空间去自我补足。

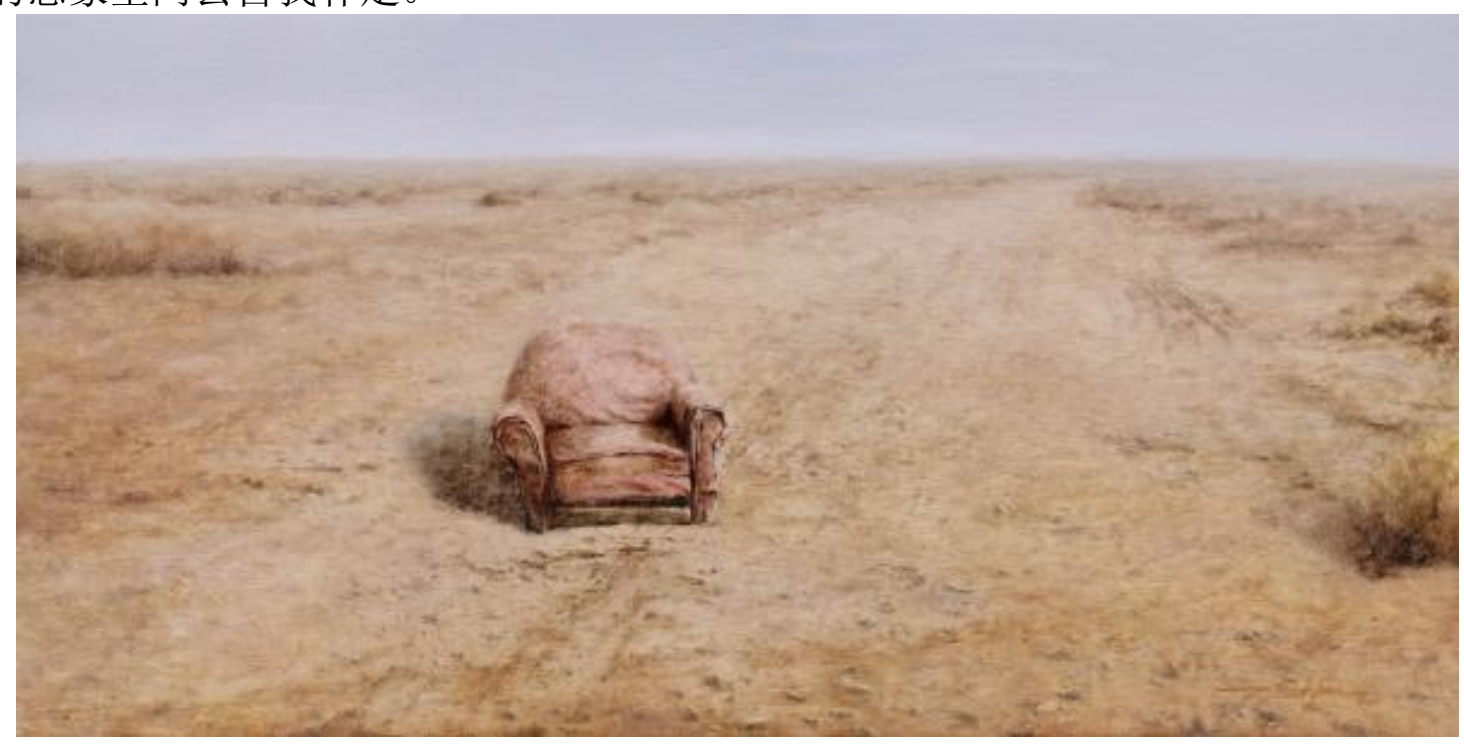

图1. 旧沙发的文化意义

\section{3. 善于构建非逻辑化的“真实”空间}

张庆慧笔下的空间都非常的 “传奇” ，事实上张庆慧油画作品所呈现的空间并非是对我们日 常熟悉生活的一种简单还原, 其更多是加入了作者对于人生、宇宙的一种思考, 也就是说张 庆慧的油画作品在现实世界几乎是不存在的, 其仅仅是画家作品的一种悖理的、异样的存在。 油画作品《被云带走的往事》也是如此, 在这幅作品中我们不得不在极为陌生化的环境下, 在一片干枯的黄土下和废弃的沙发突然的相遇到, 这种极为偶然的邂逅首先给我们视觉感官 上的唐突, 但是当我们平复心情, 我们会发现这看似荒谬的黄土和废弃的沙发其实蕴含着深 刻的含义。沙发上面虽然没有人，但是我们还是依稀的感受到一个老者在叙述着如歌的往事。 《被云带走的往事》创作上主要采用了 “计白当黑”、“虚实相生” 的表现手法, 清代邓石 如称 “字画疏处可走马, 密处不使透风, 常计白当黑, 奇趣乃出。”画面中北方特点的荒野 几乎占据了画面的全部，是 “白”、“虚”处，一张老式的单人沙发，是 “黑”、“实”处。 “疏至极则成白, 密至极则呈黑” , 画面 “布白. 计白为黑” 是以虚应实, 创造出一种空灵之 美。以虚空处见深远, 传递丰盈。这种布白就像是在一片混沌不清的世界之中一道亮光, 给 人一种清新, 顺畅的感觉。就像是在枝繁叶茂的深林之中, 因大大小小的形状各异的空白而 
形成的一种烟云变化的状态, 给人虚幻缥组, 仿佛置身于幻境之中的感觉。画面 “实” 处虽 只是一个孤立的单人沙发, 但因 “虚” 的衬托而更为突出, 所谓 “以一当十”、“以少用多”。 这种画面更能达到 “无声胜有声” 的艺术效果, 使得画面具有一种全新的境界。作品以我们熟 悉的生活用品做为绘画的主题, 其悉心挖掘蕴藏在日常生活中那些永恒的心灵话题。张庆慧 的作品没有荡气回肠, 轰轰烈烈的场景, 但是却耐人寻味, 动人心弦。

《被云带走的往事》里面沙发在此刻已经不是我们熟悉简单的生活用品。事实上在这里这个 看似废弃的沙发其实更像是一种文化符号, 是一种精神寓指。由沙发介入黄土背景制作, 使 得画面强化了主体与环境的对立关系, 激发了物象的矛盾冲突。画面平和的外表下由此而显 得暗潮涌动、富有张力。在这辽阔无垠的黄土地带, 我们曾经忽视的风景, 在画家笔下成了 情感抒发的重要载体。在这里实空间的置换颠覆了人们正常的视觉经验, 更是消解了日常家 居的传统意义。这使得画面更多了几分荒诞和非现实意味。正是艺术家这种有意无意的疏离 现实，使我们对画面深层意义的探讨有了更多的可能性。

\section{4. 个体性的批判和反思}

生活是人类社会生活的一个部分, 也是自然生活的一个重要组成元素, 离开大自然人类的家 居生活就是所谓的空谈。为此油画创作上一定要秉承自然的创作态度, 不做作不虚伪, 要多 从自然界汲取有益的营养。事实上油画创作理念也是最大化体现人与自然的和谐之美, 以便 使得画面更具一种观赏感受。在大自然中不仅有郁郁苟苟的植物, 还有形形色色的动物, 正 是这些众多生物组成使得人类社会更为丰富多彩。人与自然的和谐不仅要维护健康的生态系 统, 也要其她动物和谐相处。

细致的观看张庆慧的作品展示了一种日常现实的 “混搭” 场景, 把日常的物无隐私的置换与 自然之景, 例如《被云带走的往事》就是将一个废弃的沙发搁置在荒凉的黄土上, 这样非常 自我化的一种情景设置很容易让人们产生一种无限的遐想, 使得画面更具有一种隐喻性。张 庆慧 “隐喻” 不是一种简单地对客观世界的一种模仿与还原, 也不是作者主观世界的一种臆 断。而是一种主观世界与客观世界的统一, 是艺术家们根据自己的阅历对于客观世界的一种 认知。是一种 “天人合一”与 “物我融一” 的绘画境界, 艺术家们追求崇高理想的体现 从张庆慧《被云带走的往事》我们不难发现, 作为一名 “新锐” 画家, 张庆自慧极为重视通 过所谓 “悖理” 的画面来传达一种对于生活与生命的感悟。在黄土之中赫然出现一个破旧的 沙发, 这个画面非常有突兀感, 而且画面本身似乎也并不唯美。但是当观众整理下心情, 用 心去解读这幅作品之后, 我们会发现这看似空旷的黄土地其实承载着悠悠岁月的感慨。我们 似乎可以 “看到”一个老者在向我们讲述如歌的往事, 讲述这片黄土地的过往。黄土地依然 是黄土地, 依然没有绿色的充盈, 在时光的过往中人类在忙什么? 为什么我们的家园依然这 样的荒芜, 难道在当下的现实环境中, 难道一切内在精神都成了假象? 艺术的真实就是对这 种假象进行个体性的批判和反思。因此对个体经验的从新解读是剖析社会现实的重要一个环 节，这种现实的真实性是一种新的观念和新的艺术视觉体验。

\section{5. 追求一种 “与世界共在” 的愿望}

绘画的构图同样也是来源生活, 在生活的点滴中向我们传达着油画之美, 这种生活的构图虽 然经过了一些适当夸张与艺术处理, 但是我们在画面观感上一点都不觉得别扭, 反而有一种 舒适之感。张庆慧在绘画上并不要求具有强烈的视觉冲击力, 主要是通过生活常态化场面的 艺术加工，带给我们更多美的享受和艺术的启迪。

对于绘画创作张庆慧有一种内在诉求却在她的作品中得以保留、延续, 那就是 “与世界共在” 的欲望。“与世界共在” 是海德格尔的现象学术语, 冒着 “吊书袋子” 的危险, 我使用这一 术语来表述庆慧作品中与其一致的立场。在海德格尔看来, 人的原初状态是与世界共在的, 但所谓的理性、文明、技术却使得人与世界相分离, 由此今天的人们难以真正地认识世界, 
异化成了当代人的普遍状态。张庆慧的画面中，人们日常生活中的物件全被 “搬” 到了室外 “世界”之中, 这世界不仅仅是草地、湖泊、荒原, 也是城市、公路; 既是自然世界, 也包 括人们身处其中的人工世界。去除遮蔽在庆慧的作品中成为一贯的母题：在画面中，屋墙几 乎都不在了, 日常生活的物件向室外的世界 “敞开” , 人们重回世界怀抱。从建筑学的角度 来看, 屋墙的意义在于遮风挡雨, 在于寻求安全, 但它在客观上又阻隔了人与世界的关系, 破坏了人与世界原初的共生关系。当然, 屋墙不过是一种象征修辞, 它表征着一切阻隔人与 世界的文化产物：建筑、习俗、制度、理性。张庆慧的作品正是提供给人们这样一种想象, 提供给人们这样一种移情方式。

在《被云带走的往事》将黄土和废弃的沙发进行景观并置, 这种与众不同的绘画方法很容易 让观者对于这幅油画作品产生一种超现实主义的荒诞感。《被云带走的往事》中那个褐色沙 发给人的印象极为深刻。事实上张庆慧可以对画面进行这样处理, 将这个毫无生机的沙发安 置在荒芜的黄土之地, 使得废弃的沙发更多了一种遗弃之感, 这种荒诞化处理则似乎是在进 行审美层面的反思。画面让人产生一种莫名的焦虑, 这焦虑也许正来自于人与世界的分离, 来自于无法回复到本真状态的放逐感。

\section{6. 色彩文化体现一种虚实的意境}

色彩文化是一个民族极为重要的文化组成项目，对于一个民族文化的发展会产生极为深远的 影响。在人类不同的发展阶段、不同的地域色彩文化的内涵也有着极大的差异。客观地讲, 色彩文化会随着主流文化的发展而有所变迁，为此不同民族最终会形成不同的色彩文化与色 彩理念, 中国色彩文化主要体现在独具特色的五行上。这五色是白、青、黑、赤、黄五色, 分别对应着五行中的金、木、水、火、土。而五行所具有的深遂含义，并将其五行思想和色 彩有机的结合起来, 赋予了这五行色彩特殊的含义, 也就是我们现在所说的五行观。在这种 传统五行观的影响下, “五色” 成了中国人对于色彩认知和思维的本源, 而在随后的岁月里。 中华民族的思想、文化、政治制度以及生活起居和艺术文化都深受五色观的影响。 我们如今经常提及的词语，白金、黄土、赤火就很好的反映出传统五色观的深遂文化内涵。 中国传统绘画强调 “五色论” , 并将青、赤、黄、白、黑这五色与五行相对, 并赋予这五色 高度的象征与哲学含义。

《被云带走的往事》在绘画上虽然不以光影突出沙发的特色和个性, 但是画面的色彩感不会 显得单一，依然有着丰富的色彩对比。这是因为画家在创作中，对于沙发以及背景的色彩进 行了细致的强化处理, 红褐色的沙发、黄色的地面、淡蓝色的天空, 三种颜色的结合更能表 达出张庆慧自身内心的情感表达, 这是基于对画面的平光的处理。《被云带走的往事》画面 更倾向固有色和较纯色调的运用，作品色彩单纯、响亮、鲜明、富有感染力。 纵观张庆慧在画面色彩的运用, 也是基于这五种固有颜色上进行创作, 着重体现一种高雅、 明快与素朴的色调, 具有浓郁的中国传统文化的审美意蕴, 本文研究的《被云带走的往事》 就给人一种似幻似梦, 但却让人回味不已的艺术境界, 这种虚实相交的画面感, 使得张庆慧 油画作品寓意无穷。

\section{7. 结论}

以上是本人以《被云带走的往事》为例对张庆慧油画作品的艺术特点进行的论述, 通过上文 的论述我们可以看出张庆慧在艺术领域的巨大成就在于充分表现标本的独立形象和通过运用 个性色彩去表现自身的思想情感, 就如同将图形语言化、符号化一样, 是用特定的色彩作为 自己的语言进行内省式的精神探索。这种个性色彩的运用，使得画面中的色调具有一种隐喻 的表达, 最为突出之处在于情感与个性的有机结合, 让自己的作品更具当代虚与实的美感。 张庆慧的作品是以生活中最简单的题材为基础的, 生活味强, 她是把现实生活与自己的想象 力联系起来表现的。通过张庆慧油画作品的艺术特点, 我们可以发现她的作品感染力非常强, 
且能给观者很强的触动, 这是她的作品给人带来的现实与自然最真实的情感表达以及对生活 的真正的爱, 因此其作品的研究是具有现实意义的。

\section{References}

[1]. Lin Yuqing. Study on Symbolization of Chinese oil painting [D]. Hebei Normal University, 2017.

[2]. Mao Wenyi. Chinese oil painting and regional culture [D]. Soochow University, 2016.

[3]. Dong Haipeng. Thinking on the current situation of Chinese oil painting in 1990s [D]. Shaanxi Normal University, 2015.

[4]. A Jinpeng. The development and aesthetic value of contemporary Chinese oil painting language [D]. Chongqing University, 2012.

[5]. Xie Lin. The current situation and thinking of contemporary Chinese oil painting -- the value orientation of Chinese oil paintings from the Tenth National Art Exhibition [J]. Journal of the Art Academy of the PLA, 2005 (03) 